\title{
Influenza virus infections among a sample of hospital attendees in Ragama, Sri Lanka
}

\author{
K V H K K Perera ${ }^{1}$, K H Chan ${ }^{2}$, E Ma² $^{2}$ J S M Peiris ${ }^{2}$ \\ (Index words: Influenza A and B, seasonality, rainfall)
}

\begin{abstract}
Objectives This study was carried out to define the types of influenza viruses circulating among humans and to understand the seasonality of influenza virus activity. Such information is essential for deciding on influenza vaccination strategy and on the appropriate time for delivering influenza vaccination, if such a vaccination policy was decided to be a priority.

Method During the period July 2003 - August 2004, 300 nasopharyngeal aspirates (NPA) were obtained from a systematic sample of patients reported to Out-patient Department, Colombo North Teaching Hospital, Ragama with $\leq 4$ days history of acute respiratory tract infection (ARTI). The clinical signs and symptoms of the patients were prospectively recorded. Isolation of the influenza virus was carried out by inoculating in Madin Darby Canine Kidney cell line (MDCK). The isolates were identified by immunofluorescence assay and characterised by haemagglutination inhibition test. RT-PCR was carried out on all NPA samples. Genetic sequencing and phylogenetic analysis of the haemagglutinin gene of representative viruses were carried out.
\end{abstract}

Results Twenty three influenza A and nine influenza B viruses were isolated by cell culture methods. Influenza A H3N2 Panama/2000/99-like viruses were isolated in $8 \%$ of patients with ARTI and influenza B/Sichuan/ $379 / 99-l i k e$ viruses were isolated in 3\%. Twenty eight influenza A virus infections were identified by the RT-PCR method. Phylogenetic analysis was carried out with data from other H3-subtype viruses isolated worldwide. The Sri Lanka viruses are antigenically and genetically similar to those in the northern and southern hemispheres.

Conclusions Influenza viruses circulate at different times of the year and is the aetiological agent causing $11 \%$ of all ARTI. Influenza activity corresponded to a peak in rainfall; however the correlation of influenza virus activity with rainfall is not invariable. The Sri Lankan isolates of 2003-4 were genetically related to the influenza A viruses circulating around the globe.

\section{Introduction}

Influenza is a contagious, acute, self limited febrile respiratory illness of global importance caused by three types of influenza virus, types A, B and C $[1,2,3]$. Influenza A viruses are known to cause frequent annual epidemics and periodic pandemics. Type B influenza virus shows antigenic drift as with influenza $\mathrm{A}$. There is no animal reservoir of influenza B viruses. Therefore there are no pandemics of influenza $B$. Type $C$ influenza virus is antigenically stable and causes asymptomatic infection of mild upper respiratory illness. For both type $\mathrm{B}$ and $\mathrm{C}$ viruses there is only one subtype of HA and NA $[4,5]$.

About $20 \%$ of children and $5 \%$ of adults world-wide develop symptomatic influenza A and B each year [6]. Influenza is a typically winter disease in temperate regions, it has a broader or even year-round seasonality in tropical regions, with peak virus isolation during periods of high rainfall $[1,2]$.

Influenza virus was first isolated in Sri Lanka (then Ceylon) during the pandemic of "Hong Kong flu" H3N2 in 1969 [7,8]. Viral isolation and serological studies carried out at the Colombo South virus laboratory revealed evidence of acute infection with $\mathrm{H} 3 \mathrm{~N} 2$ subtype of influenza virus in years 1988 and 1989. H3N2 and H1N1 serotypes of influenza viruses were isolated from time to time since 1998. However, there has been no systematic year round study of influenza seasonality reported from Sri Lanka since that reported during the pandemic of 1968 . We report a systematic study on influenza like illness carried out in Ragama, Sri Lanka over a 13 month period, 2003-2004.

\section{Method}

Study Population: Patients attending the OPD (Outpatients Department) of the Colombo North Teaching Hospital, Ragama, for the treatment of acute upper respiratory tract infection of 1-4 days duration were included in the study. Nasopharyngeal aspirates (NPA) were collected from the patients (irrespective of age and sex) on Mondays and Fridays during the period from July 2003 August 2004. Patients presenting at the OPD between 8.00 a.m.-12 noon during the two days (Monday and Friday) of the week were recruited to the study. Eight common presenting symptoms of influenza infection were recorded on all patients. Informed written consent was obtained from patients, and in the case of children, from parents or guardian. The Ethical Review Committee of the Faculty of Medicine, University of Kelaniya approved the study.

${ }^{1}$ Department of Medical Microbiology, University of Kelaniya, Kelaniya, Sri Lanka, ${ }^{2}$ Department of Microbiology, Faculty of Medicine, University of Hong Kong, Hong Kong - SAR.

Correspondence: KVHKKP, e-mail <harshaperera@gmail.com>. Received 19 August and revised version accepted 26 December 2009. Competing interests: none declared. 
Sample collection: NPA were obtained by inserting a fine (gauge 6 and 7 for children and adults respectively) catheter (Pacific Medicals, Taiwan) to the posterior nasopharynx via the external nares and applying suction pressure. After aspirating secretions via both nostrils, the catheter was inserted into a bijou bottle containing $2 \mathrm{ml}$ of viral transport medium (VTM) and the remaining secretions in the catheter washed into the mucus trap. The secretions with the VTM was kept at $+4{ }^{\circ} \mathrm{C}$ until transported to the laboratory. VTM used was Earle's buffered saline with 5\% bovine albumin, antibiotics and antifungal agents [vancomycin $(100 \mu \mathrm{g} / \mathrm{ml})$, amikacin $(30 \mu \mathrm{g} / \mathrm{ml})$, nystatin $(40 \mu \mathrm{g} / \mathrm{ml})]$.

Virus isolation by cell culture: Confluent monolayers of MDCK cells grown in tissue culture tubes were washed in serum free medium and $200 \mu \mathrm{l}$ of the specimen in VTM was inoculated into the cell culture tubes and incubated at $37^{\circ} \mathrm{C}$ for one hour for absorption. Low passage level $(<15$ passage) MDCK cells were used for this study and were obtained from the University of Hong Kong. After one hour incubation, $1 \mathrm{ml}$ of serum free medium supplemented with $2 \mu \mathrm{g} / \mathrm{ml}$ 1-Tosylanide-20 phenylethyl chloromethyl ketone (TPCK) treated trypsin was added and the tubes were incubated at $37^{\circ} \mathrm{C}$ with $\mathrm{CO}_{2}$. On the following day, medium was removed and $1 \mathrm{ml}$ of fresh serum free medium supplemented with $2 \mu \mathrm{g} / \mathrm{ml}$ of TCPK treated trypsin was added and the tubes incubated for a further 7 days at $37^{\circ} \mathrm{C}$.

The cells were observed daily for cytopathic effect (CPE). Cells were scraped and cell smears were tested by immunofluorescence using DAKO Imagen Influenza A and B antibody Dako Cytomation ${ }^{\mathrm{TM}}$ (Cambridgeshire, England) to detect influenza viruses. Haemagglutination inhibition tests were done to subtype and antigenically characterise the isolate using reference antisera and antigens provided by World Health Organization (WHO) Collaborating Center at the Center for Diseases Control and Prevention, Atlanta. USA. The haemagglutination inhibition test and the neuraminidase inhibition test were carried out according to the animal influenza training manual issued by the WHO [9].

Virus nucleotide detection by RT-PCR: Viral extraction was carried out as instructions laid down by the manufacturer (QIAGEN GmbH, Hilden, Germany). Extracted viral RNA was tested by reverse transcriptionpolymerase chain reaction (RT-PCR). Random primers (Invitrogen, Life Technology, Carlsbad, CA) and of Superscript II Rnase H Reverse Transcriptase (Invitrogen, Life Technology) was used to make complementary DNA (cDNA). The cDNA was used for PCR amplification reaction with $1 \mu \mathrm{M}$ each of two primers (sense 170801E -5, 'TTCTAACCGAGGTCGAAACG 3' and anti-sense 170801F-5" 'GGC ATTTTGGACAAAKCGTCT 3') corresponding to the sequence of the $\mathrm{M}$ gene of influenza A virus. The size of M gene RT-PCR product was $243 \mathrm{bp}$.
Sequencing of viral genome: Viral RNA was extracted from the MDCK cell cultures using QIAamp viral RNA mini kit (Qiagen, Chatsworth CA) and a one-step RT-PCR was performed using Superscript TM III one-step RT-PCR with Platinum Taq (Invitrogen). All PCR products were purified by QIAquick PCR purification kit (Qiagen). Sequencing was performed as described earlier [10]. Phylogenetic tree was set based on the gene sequencing results.

\section{Results}

A total of 300 nasopharyngeal aspirate specimens were collected during the 13 month period (Figure 1). Of the patients with ARTI (acute respiratory tract infection) recruited to the study, $199(66.3 \%)$ were less than 15 years of age and $74(25 \%)$ were less than 5 years of age (Table 1$)$. The mean duration of the illness in this study group was 2.5 days, most of the patients $(115 ; 38 \%)$ presented to the hospital on the 3rd day of illness. Eight presenting symptoms were assessed on each patient. Two hundred and fifty seven (257/300) patients presented with cough, 235 had fever and 231 had nasal stuffiness. Rest of the frequency of presenting symptoms were nasal stuffiness $(89 / 300)$, headache $(88 / 300)$, myalgia $(82 / 300)$, sore throat $(69 / 300)$ and chills $(38 / 300)$ in descending order.

Influenza virus was isolated from 33 specimens by cell culture methods, 24 were identified as influenza A and 9 were influenza B. All nine influenza B viruses and 14 influenza A viruses were isolated in the first passage of the specimen in cell culture. The remaining 10 influenza A viruses were isolated by one "blind passage" in cell culture. The median time to development of virus CPE in those that were isolated in the first passage was 6 days for influenza A and 3 days for influenza B. Overall, influenza $A$ and influenza B viruses were isolated from $8 \%(n=24)$ and $3.0 \%(n=9)$ of the specimens respectively. Thirteen out of 24 (13/24) influenza A and seven out of nine (7/9) influenza B were isolated from patients less than 15 years of age (Table 1). All influenza A isolates were subtyped using the WHO reference antisera revealed these viruses to be subtype H3N2 and they were antigenically closely related to A/Panama/2000/99 (H3). All influenza B isolates were closely related with antisera to B/Sichuan/379/99.

All 300 specimens were also tested for influenza A by RT-PCR. Twenty eight positives were obtained, including all 24 culture positive specimens and additional positive specimens.

Influenza A virus activity peaked during May-July 2004 with a minor peak of virus activity during October December 2003 (Figure 1). Eighteen (55\%) of the 33 influenza viruses were isolated during the two month period, May-July 2004. There were three peaks of ARTI during the period under study (Figure 1) but only one of these (May-July 2004) correlated with a peak of influenza (Figure 1 and 2). However, even at this time, only 5.3\% of ARI cases had influenza virus infection confirmed virologically. 
Table 1. Age distribution and the pattern of the influenza isolates

\begin{tabular}{cccc}
\hline Age Range & Number of patients & Influenza A isolates & Influenza B isolates \\
\hline $0-5$ & 111 & 6 & 4 \\
$6-10$ & 60 & 4 & 2 \\
$11-15$ & 28 & 3 & 0 \\
$16-20$ & 20 & 2 & 0 \\
$20-25$ & 23 & 3 & 1 \\
$26-30$ & 12 & 0 & 0 \\
$31-35$ & 11 & 3 & 0 \\
$36-40$ & 10 & 1 & 1 \\
$>40$ & 25 & 2 & 1 \\
Total & 300 & 24 & 9
\end{tabular}

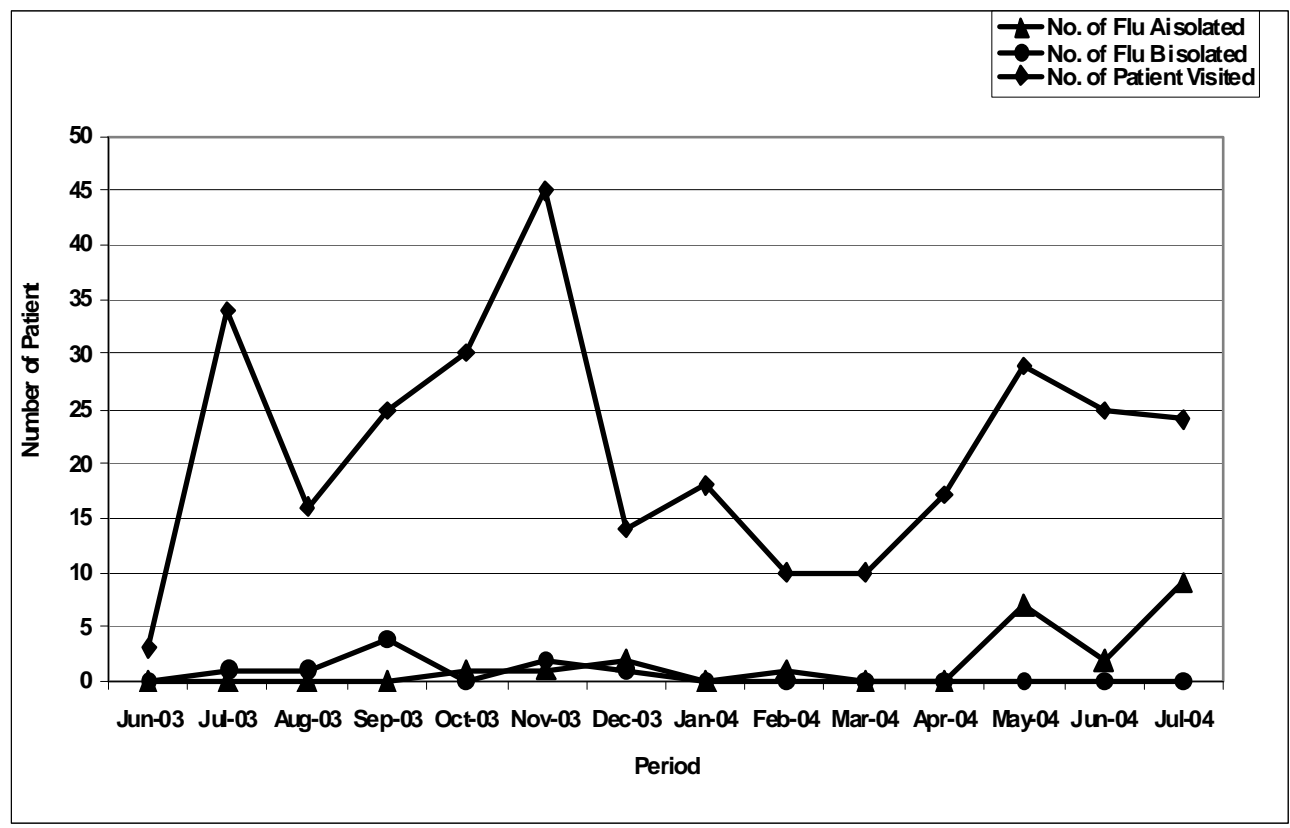

Figure 1. Number of patients visited the OPD and distribution of flu isolates.

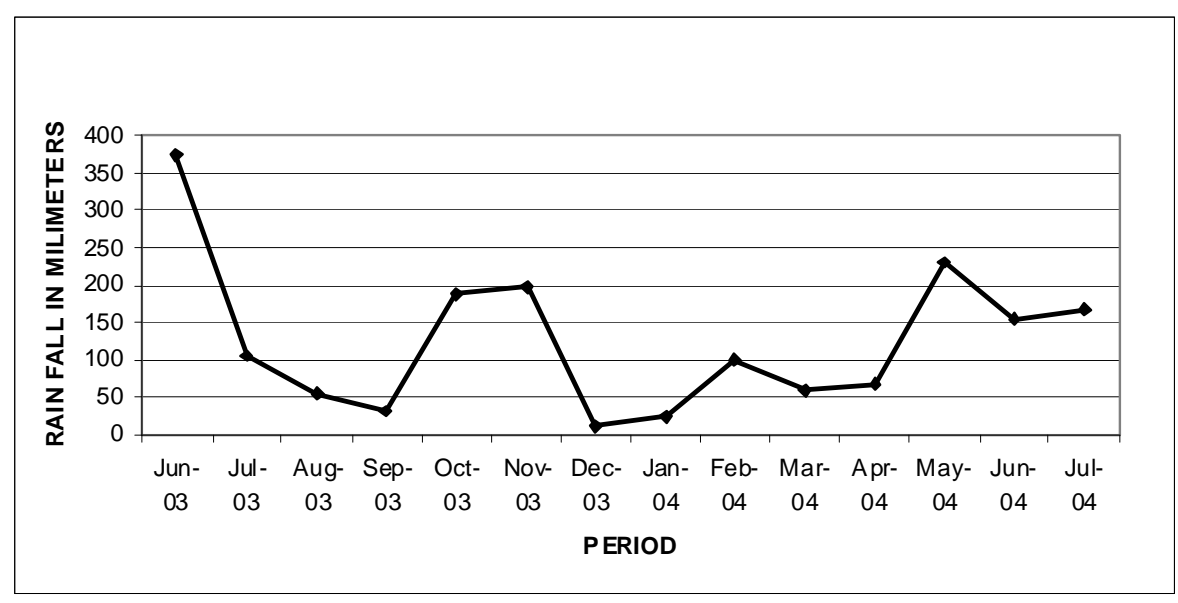

Figure 2. Rainfall figures of the years 2003-2004 in Ragama area in millimeters. (Courtesy of Department of Meteorology, Colombo, Sri Lanka.) 
The rainfall data during this period is shown in figure 2. There are three peaks of rainfall during the study period, one around July 2003, from October-November 2003 and May-June 2004.

The virus haemagglutinin (HA) of three influenza A virus was partially genetically sequenced and a phylogenetic tree was constructed together with other H3-subtype viruses isolated worldwide. The results show that the Sri Lankan isolates of 2003-4 were genetically closely related to the other influenza A viruses circulating around the globe during the study period, including those from Japan, USA, Australia and New Zealand.

\section{Discussion}

The results from this study show that influenza viruses circulate at different times of the year and is the aetiological agent causing $11 \%$ of all ARTI. The largest peak of influenza A virus activity during May-June 2004 corresponded to a peak in rainfall, however the correlation of influenza virus activity with rainfall is not invariable. The peaks of influenza A did not correlate with that of influenza B. Influenza B virus activity occurred from September 2003 to December 2003 at low levels without a major peak of virus activity.

Sixty percent (60.6\%) of influenza viruses ( Influenza $\mathrm{A}$ and $\mathrm{B}$ ) were isolated from patients less than 15 years age group suggest, influenza virus infection is more prevalent among this age group.

In other tropical countries such as Singapore, influenza also tends to occur throughout the year while in
Thailand, virus activity correlates with rainfall [11-13]. Our data are based only on a single year and data from several years are needed before conclusions can be drawn on the seasonality of influenza virus activity in Sri Lanka. Such information is important before the timing of influenza vaccination for Sri Lanka is determined. We found that RT-PCR was more sensitive for the detection of influenza A than culture and $14 \%$ of influenza A positive would have been missed if only virus culture was relied upon. As we did not do RT-PCR for influenza B, we probably may have missed a proportion of influenza $B$ viruses as well.

Our phylogenetic and antigenic results (Figure 3) demonstrate that the viruses circulating in Sri Lanka were genetically and antigenically similar to those circulating elsewhere in the world [14]. Identification of prototype influenza virus for the annual influenza vaccination programme heavily depends on systematic surveillance of these viruses. Thus it is important to strengthen the influenza surveillance programme in the country. Influenza is also a significant cause of morbidity and mortality in patients of any age with underlying cardio-respiratory disease, diabetes, renal disease and those who are immunecompromised. Thus an appropriate risk assessment should be carried out for Sri Lanka in relation to other causes of morbidity and mortality to guide vaccination policy.

Financial assistance: National Science Foundation Sri Lanka grant number: RG/2003/M/05/ and National Institute of Allergy and Infectious Disease. Contract number HHSN266200700005C, University of Hong Kong, Hong Kong SAR.

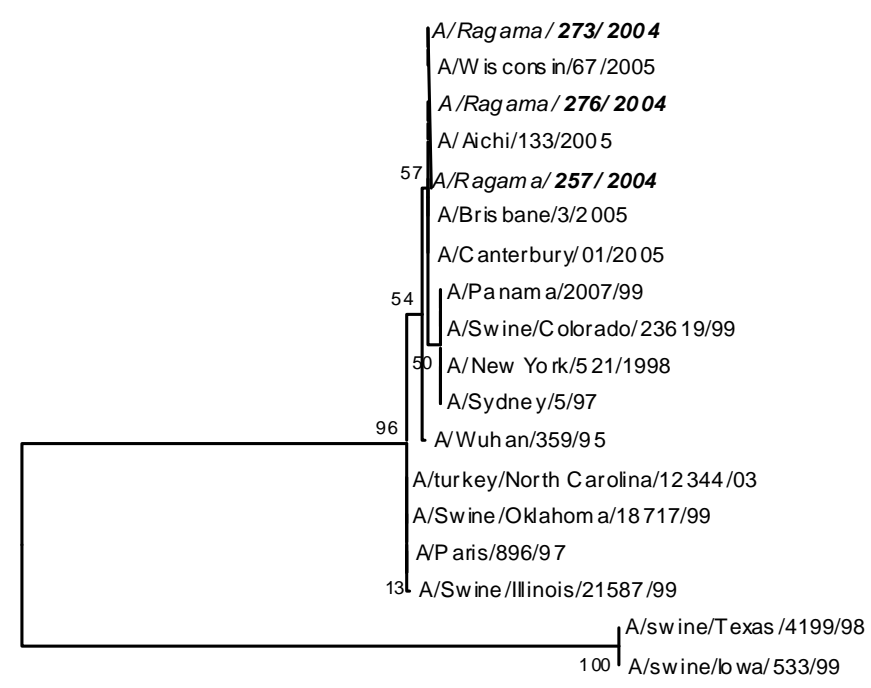

Figure 3. Phylogenic tree

Isolates given in italics are the some of the influenza viruses isolated in the study. 


\section{References}

1. Dosseh ANK, Spiegel A, Sagna MMC. Epidemiological and virological influenza survey in Dakar, Senegal 1996-1998. American Journal of Tropical Medicine and Hygiene 2000; 62: 639-43.

2. Weber MW, Mulholl, EK, Greenwood BM. Respiratory syncytial virus infection in tropical and developing countries. Tropical Medicine and International Health 1998; 3: 268-80.

3. Yang L,Wong CM, Lau EHY, et al. Synchrony of clinical and laboratory surveillance for influenza in Hong Kong PLoS ONE 2008;1:e1339. Available at www.plosone.org

4. Webster RG, Bean, WJ Gorman OT, et al. Evolution and ecology of influenza A viruses. Microbiology Reviews 1992; 56: 152-79.

5. Smith GJ, Vijaykrishna D, Bahl J, et al. Origins and evolutionary genomics of the 2009 swine-origin H1N1 influenza A epidemic (letter). Nature 2009; 459: 1122-5.

6. Turner D, Wailoo A, Nicholson K, et al. Systematic review and economic decision modeling for the prevention and treatment of influenza A and B. NICE Assessment Report 2002. Available at www.nice.org.uk/pdf/influenzaassrep.pdf

7. Playford EG, Dwyer DE. Laboratory diagnosis of influenza virus infection. Pathology 2002; 34: 115-25.

8. Mendis NM, Maheswaran K, Mendis O, et al. Epidemic of influenza in Ceylon. The Ceylon Medical Journal 1970; 15: 195-206.
9. Activities of the Institute 1988/1989. Bulletin of the Medical Research Institute of Sri Lanka 1991; 5: 49-50.

10. World Health Organization, 2000. Manual on animal influenza diagnosis and surveillance. No 2002.5. WHO Geneva, Switzerland.

11. Guan Y, Shortridge KF, Krauss S, et al. H9N2 influenza viruses possessing H5N1-like internal genomes continue to circulate in poultry in southeastern China. Journal of Virolology 2000; 74: 9372-80.

12. Chow A, Ma S, Ling AE, Chew SK. Influenza-associated deaths in tropical Singapore. Emerging Infectious Diseases 2006; 12: 114-21.

13. Simmerman JM, Uyeki TM. The burden of influenza in East and South-East Asia: A review of the English language literature. Influenza and Other Respiratory Viruses 2008; 2: 81-92.

14. Suntarattiwong $P$, Sian-nork C, Thongtipa P, et al. Influenzaassociated hospitalization in urban Thai children. Influenza and Other Respiratory Viruses 2007; 5: 177-82.

15. Guy B, Stéphanie C, Pierre D, et al. Multiplex real-time PCR assay for detection of influenza and human respiratory syncytial viruses. Journal of Clinical Microbiology 2004; 42: 45-51.

16. Ramamurthy N, Pillai LC. Gunasekaran P, et al. Influenza activity among the paediatric age group in Chennai. Indian Journal in Medical Researches 2005; 121: 776-9. 